\title{
Invariance in a class of operations related to weighted quasi-geometric means
}

\author{
Jimmy Devillet and Janusz Matkowski
}

\begin{abstract}
Let $I \subset(0, \infty)$ be an interval that is closed with respect to the multiplication. The operations $C_{f, g}: I^{2} \rightarrow I$ of the form

$$
C_{f, g}(x, y)=(f \circ g)^{-1}(f(x) \cdot g(y)),
$$

where $f, g$ are bijections of $I$ are considered. Their connections with generalized weighted quasi-geometric means is presented. It is shown that invariance question within the class of this operations leads to means of iterative type and to a problem on a composite functional equation. An application of the invariance identity to determine effectively the limit of the sequence of iterates of some generalized quasi-geometric mean-type mapping, and the form of all continuous functions which are invariant with respect to this mapping are given. The equality of two considered operations is also discussed.
\end{abstract}

\section{Introduction}

Let $X$ be a set and let $\oplus: X^{2} \rightarrow X$ be a bisymmetric operation. In [7, given bijective functions $f, g: X \rightarrow X$, the class of operations $D_{f, g}: X^{2} \rightarrow X$ defined by

$$
D_{f, g}(x, y)=(f \circ g)^{-1}(f(x) \oplus g(y)), \quad x, y \in X,
$$

where " $\circ "$ stands for the composition, was introduced and their relation with means was investigated in the case where $X \subset \mathbb{R}$ is an open real interval and $\oplus$ is the usual addition. In particular, given arbitrary bijective functions $f, g, h: X \rightarrow X$ such that $D_{f, g}$ and $D_{g, h}$ are reflexive, it was shown (see [7, Theorem 1]) that the function $D_{f \circ g, g \circ h}$ is invariant with respect to the mapping $\left(D_{f, g}, D_{g, h}\right): X^{2} \rightarrow X^{2}$, i.e. that

$$
D_{f \circ g, g \circ h} \circ\left(D_{f, g}, D_{g, h}\right)=D_{f \circ g, g \circ h} .
$$

In the present paper, given an interval $I \subset(0, \infty)$ that is closed with respect to the multiplication and bijective functions $f, g: I \rightarrow I$, we consider the class of operations $C_{f, g}: I^{2} \rightarrow I$ of the form

$$
C_{f, g}(x, y)=(f \circ g)^{-1}(f(x) \cdot g(y)),
$$

\footnotetext{
${ }^{0} 2010$ Mathematics Subject Classification. Primary: 26A18, 26E60, $39 \mathrm{~B} 12$.

Keywords and phrases: invariant functions, mean, invariant mean, reflexivity, iteration, functional equation
} 
where "." (which will be sometimes omitted) stands for the usual multiplication. (Of course $C_{f, g}$ form a subclass of the operations $D_{f, g}$.)

The similarity of $C_{f, g}$ to the generalized weighted quasi-geometric means introduced in Section 2, motivates our considerations in Section 3, where we examine conditions under which $C_{f, g}$ is a bivariable mean. The reflexivity property of every mean leads to the iterative functional equation

$$
f(g(x))=f(x) \cdot g(x), \quad x \in I,
$$

where $I=(1, \infty)$ (or $I=[1, \infty)$ ) and the functions $f$ and $g$ are unknown. Under some natural conditions, Theorem 3 , the main result of this section, says in particular that $C_{f, g}$ is a bivariable mean in $(1, \infty)$, if and only if,

$$
f=\prod_{k=0}^{\infty} g^{-k}
$$

where the infinite product $\prod_{k=0}^{\infty} g^{-k}$ of iterates of $g^{-1}$ converges uniformly on compact subsets of $(1, \infty)$; moreover $C_{f, g}=\mathcal{C}_{g}$, where

$$
\mathcal{C}_{g}(x, y):=\left(\prod_{k=0}^{\infty} g^{-k+1}\right)^{-1}\left(\prod_{k=0}^{\infty} g^{-k}(x) \cdot g(y)\right), \quad x, y>1,
$$

which justify the names: iterative type mean for $\mathcal{C}_{g}$, and iterative generator of this mean for $g$.

If $C_{f, g}=\mathcal{C}_{g}$ and $C_{g, h}=\mathcal{C}_{h}$, then, in view of [7, Theorem 1], the function $C_{f \circ g, g \circ h}$ is invariant with respect to the mean type mapping $\left(\mathcal{C}_{g}, \mathcal{C}_{h}\right)$.

On the other hand $\mathcal{C}_{g}$ (and $\mathcal{C}_{h}$ ) is a very special case of generalized weighted quasi-geometric mean $G_{\varphi, \psi}: I^{2} \rightarrow I$, of the form

$$
G_{\varphi, \psi}(x, y)=(\varphi \cdot \psi)^{-1}(\varphi(x) \cdot \psi(y)), \quad x, y \in I,
$$

where $\varphi, \psi: I \rightarrow(0, \infty)$ are continuous, of same type monotonicity, and such that $\varphi \cdot \psi$ is strictly monotonic in the interval $I$ (see Section 2). Moreover, since generalized weighted quasi-geometric means can be considered as generalized strict weighted quasi-arithmetic means (see Section 2), if $G_{\varphi, \psi}$ and $G_{\psi, \gamma}$ are two generalized weighted quasi-geometric means, then $G_{\varphi \cdot \psi, \psi \cdot \gamma}$, the mean of the same type, is a unique mean that is invariant with respect to the mean-type mapping $\left(G_{\varphi, \psi}, G_{\psi, \gamma}\right): I^{2} \rightarrow I^{2}$ (see [6]). This leads to natural and equivalent questions: is $C_{f \circ g, g \circ h}$ a mean; is the operation $C_{f \circ g, g \circ h}$ a generalized weighted quasi-geometric mean; or simply can the three operations $C_{f, g}, C_{g, h}$ and $C_{f \circ g, g \circ h}$ be means simultaneously? In Section 4 we prove that it can happen iff

$$
g=\prod_{i=0}^{\infty} h^{-i}, \quad f=\prod_{j=0}^{\infty}\left(\prod_{i=0}^{\infty} h^{-i}\right)^{-j},
$$

and $h$ satisfies "strongly" composite functional equation

$$
\left(\prod_{j=0}^{\infty}\left(\prod_{i=0}^{\infty} h^{-i}\right)^{-j+1}\right)=\prod_{k=0}^{\infty}\left(\prod_{i=0}^{\infty} h^{-i+1}\right)^{-k}
$$

where $i, j, k$ stand for the indices of iterates of the suitable functions (Theorem 4). We propose as an open problem to find the continuous solutions $h:(1, \infty) \rightarrow$ $(1, \infty)$. In illustrative Example $2\left(\right.$ with $h(x)=x^{\frac{1}{w}}$ and $\left.w \in(0,1)\right)$ we get $\mathcal{C}_{g}(x, y)=$ 
$x^{1-w} y^{w}, \mathcal{C}_{h}(x, y)=x^{w} y^{1-w}$, and $C_{f \circ g, g \circ h}=\mathcal{G}^{2 w(1-w)}$, where $\mathcal{G}(x, y):=\sqrt{x y}$ is the geometric mean, which shows $C_{f \circ g, g \circ h}$ need not be a mean.

In section 5 we apply the invariance identity to determine effectively the limit of the sequence of iterates of some generalized weighted quasi-geometric mean-type mappings, as well as, to find the form of all continuous functions which are invariant with respect to these mappings.

In section 6 the equality of two considered operations is discussed.

\section{Generalized weighted quasi-geometric means}

Let $I \subset \mathbb{R}$ be an interval. Recall that a function $M: I^{2} \rightarrow \mathbb{R}$ is said to be a (bivariable) mean if it is internal, i.e.

$$
\min (x, y) \leq M(x, y) \leq \max (x, y), \quad x, y \in I,
$$

and a (bivariable) strict mean if it is a mean and these inequalities are sharp for all $x \neq y$.

Recall some well known properties of means.

REMARK 1. The following three conditions are equivalent:

(i) a function $M: I^{2} \rightarrow \mathbb{R}$ is a mean in an interval $I$;

(ii) $M\left(J^{2}\right) \subset J$ for every subinterval $J \subset I$;

(iii) $M\left(J^{2}\right)=J$ for every subinterval $J \subset I$.

REMARK 2. (i) If $M$ is a mean in $I$, then $M$ is reflexive, i.e.

$$
M(x, x)=x, \quad x \in I,
$$

(but the converse implication fails).

(ii) If a function $M: I^{2} \rightarrow \mathbb{R}$ is reflexive and (strictly) increasing in each variable then it is a (strict) mean in $I$.

Among many classes of means, one of the most popular is the family of quasiarithmetic means, which have the following generalization.

If the functions $\varphi, \psi: I \rightarrow \mathbb{R}$ are continuous and both strictly increasing or both strictly decreasing then the function $A_{\varphi, \psi}: I^{2} \rightarrow I$ defined by

$$
A_{\varphi, \psi}(x, y):=(\varphi+\psi)^{-1}(\varphi(x)+\psi(y)),
$$

is a strict mean, called a generalized strict weighted quasi-arithmetic mean in $I$ (see [4]), where (with a little modified notation) the following result is proved:

TheOREM 1. Suppose that $A_{\varphi, \psi}$ and $A_{\Phi, \Psi}$ are generalized strict weighted quasiarithmetic means in $I$. Then $A_{\Phi, \Psi}=A_{\varphi, \psi}$ if and only if there exist $\alpha, \beta, \gamma \in \mathbb{R}$, $\alpha \neq 0$, such that

$$
\Phi(x)=\alpha \varphi(x)+\beta, \quad \Psi(x)=\alpha \psi(x)+\gamma, \quad x \in I .
$$

If the functions $f, g: I \rightarrow(0, \infty)$ are continuous and both strictly increasing or both strictly decreasing then, similarly as in the case $A_{\varphi, \psi}$ one can show that the function $G_{f, g}: I^{2} \rightarrow I$ defined by

$$
G_{f, g}(x, y):=(f \cdot g)^{-1}(f(x) g(y)),
$$

is a strict mean (Here"." stands for the usual multiplication of functions: $(f \cdot g)(x):=$ $f(x) g(x)$ for every $x \in I$, and we omit writing it later). In the sequel, $G_{f, g}$ is called a generalized weighted quasi-geometric mean in $I$ of generators $f$ and $g$. 
The following remark, which is easy to verify, provides a one-to-one correspondence between the class of generalized strict weighted quasi-arithmetic means and the class of generalized weighted quasi-geometric means.

REMARK 3. For all continuous and of the same type strict monotonicity functions $f, g: I \rightarrow(0, \infty)$,

$$
G_{f, g}=A_{\log \circ f, \log \circ g}
$$

and, for all continuous and of the same type strict monotonicty functions $\varphi, \psi: I \rightarrow$ $\mathbb{R}$,

$$
A_{\varphi, \psi}=G_{\exp \circ \phi, \exp \circ \psi}
$$

Applying Theorem 1 we obtain the following

THEOREM 2. Suppose that $G_{f, g}$ and $G_{\bar{f}, \bar{g}}$ are generalized weighted quasi-geometric means in $I$. Then

$$
G_{\bar{f}, \bar{g}}=G_{f, g}
$$

if, and only if, there exist $a \neq 0, b, c>0$ such that

$$
\bar{f}(x)=b[f(x)]^{a}, \quad \bar{g}(x)=c[g(x)]^{a}, \quad x \in I .
$$

Proof. Setting $\varphi=\log \circ f, \psi=\log \circ g, \Phi=\log \circ \bar{f}, \Psi=\log \circ \bar{g}$, we see that $G_{f, g}=G_{\bar{f}, \bar{g}}$ iff $A_{\Phi, \Psi}=A_{\varphi, \psi}$, and, in view of Theorem 1, iff there are $\alpha, \beta, \gamma \in \mathbb{R}$, $\alpha \neq 0$, such that

$$
\Phi(x)=\alpha \varphi(x)+\beta, \quad \Psi(x)=\alpha \psi(x)+\gamma, \quad x \in I,
$$

that is, iff

$$
\begin{gathered}
\log \circ \bar{f}(x)=\alpha \log \circ f(x)+\beta, \quad \log \circ \bar{g}(x)=\alpha \log \circ g(x)+\gamma, \quad x \in I, \\
\bar{f}(x)=e^{\beta}[f(x)]^{\alpha}, \quad \bar{g}(x)=e^{\gamma}[g(x)]^{\alpha}, \quad x \in I .
\end{gathered}
$$

Setting $a=\alpha, b=e^{\beta}, c=e^{\gamma}$, we obtain the result.

\section{Operation $C_{f, g}$ and means}

Let $I \subset(0, \infty)$ be an interval that is closed with respect to the multiplication and let $f, g: I \rightarrow I$ be bijective functions. Then, the two-variable function $C_{f, g}$ : $I^{2} \rightarrow I$ given by

$$
C_{f, g}(x, y):=(f \circ g)^{-1}(f(x) g(y)), \quad x, y \in I,
$$

is correctly defined.

In this section we examine when $C_{f, g}$ is a mean.

LEMma 1. If $C_{f, g}: I^{2} \rightarrow I$ defined by (2) is symmetric then $g=c f$ for some real constant $c \in I, c \neq 0$; if moreover $C_{f, g}$ is a mean, then

$$
C_{f, g}(x, y)=\mathcal{G}(x, y), \quad x, y \in I,
$$

where $\mathcal{G}(x, y):=\sqrt{x y}$ is the geometric mean. 
Proof. By (2), the symmetry of $C_{f, g}$ implies that $f(x) g(y)=f(y) g(x)$ for all $x, y \in I$, that is $\frac{g(x)}{f(x)}=\frac{g(y)}{f(y)}$ for all $x, y \in I$, whence $\frac{g}{f}=c$ for some positive real constant $c$, which proves the first result. If $C_{f, g}$ is a mean, making use of (2) and the reflexivity of $C_{f, g}$, we get $f(c f(x))=f(x)(c f(x))$ for all $x \in I$ or, equivalently, $c f(c f(x))=(c f(x))(c f(x))$ for all $x \in I$, whence, in view of the equality $g=c f$, we get $g(g(x))=(g(x))^{2}$ for all $x \in I$. Since $g$ is bijective, it follows that $g(x)=x^{2}$ for all $x \in I$. Now it is easy to get that $f(x)=x^{2}$ for all $x \in I$. Consequently, making use of of (2), we obtain, for all $x, y \in I$,

$$
C_{f, g}(x, y)=\sqrt{\sqrt{x^{2} y^{2}}}=\sqrt{x y}=\mathcal{G}(x, y),
$$

which completes the proof.

Since every mean is reflexive (Remark 2(i)), we first consider conditions for reflexivity of $C_{f, g}$. We begin with

Lemma 2. Let I be a nontrivial interval that is closed with respect to the multiplication. Assume that $f, g: I \rightarrow I$ are bijective functions such that $C_{f, g}$ defined by (2) is reflexive, i.e. that

$$
(f \circ g)^{-1}(f(x) g(x))=x, \quad x \in I .
$$

If $g$ has a fixpoint $x_{0} \in I$, then $x_{0}=1$; in particular 1 must belong to $I$;

if moreover $g$ is continuous and $I \subset[1, \infty)$, then $I=[1, \infty) ; g$ is strictly increasing and either

$$
1<g(x)<x, \quad x \in(1, \infty)
$$

or

$$
g(x)>x, \quad x \in(1, \infty) .
$$

Proof. From (3) we have

$$
f(g(x))=f(x) g(x), \quad x \in I .
$$

Thus, if $g\left(x_{0}\right)=x_{0}$ for some $x_{0} \in I$, then $f\left(x_{0}\right)=f\left(x_{0}\right) x_{0}$, so $x_{0}=1$. Hence, if $I \subset[1, \infty)$ then, as $I$ is nontrivial and closed with respect to the multiplication, it must be of the form $[1, \infty)$. Since $g$ has no fixpoints in $(1, \infty)$, the continuity of $g$ implies it must be increasing and either $1<g(x)<x$ for all $x \in I$, or $g(x)>x$ for all $x \in I$.

This lemma justifies the assumption that $I=(1, \infty)$ in our considerations of reflexivity of $C_{f, g}$.

Proposition 1. Let $g:(1, \infty) \rightarrow(1, \infty)$ be injective continuous and such that

$$
1<g(x)<x, \quad x>1 .
$$

Then there is no continuous function $f:(1, \infty) \rightarrow(1, \infty)$ satisfying equation

$$
f(g(x))=f(x) g(x), \quad x \in(1, \infty) ;
$$

in particular, there is no injective continuous function $f:(1, \infty) \rightarrow(1, \infty)$ such that $C_{f, g}$ is reflexive in $(1, \infty)$. 
Proof. The continuity of $g$ and condition (4) imply that

$$
\lim _{n \rightarrow \infty} g^{n}(x)=1, \quad x>1,
$$

where $g^{n}$ denotes the $n$th iterate of $g$.

Assume that there is a continuous and strictly increasing function $f:(1, \infty) \rightarrow$ $(1, \infty)$ satisfying $(5)$. From $(5)$, by induction we get

$$
f\left(g^{n}(x)\right)=f(x) \prod_{k=1}^{n} g^{k}(x), \quad x \in(1, \infty), n \in \mathbb{N} .
$$

Since $f$ is nonnegative and increasing, it has a finite right-hand side limit at 1 , denoted by $f(1+)$. Letting here $n \rightarrow \infty$, we obtain

$$
f(1+)=f(x) \prod_{k=1}^{\infty} g^{k}(x), \quad x \in(1, \infty),
$$

that is a contradiction, as the left side is real constant and the right side is either strictly increasing or $\infty$.

Proposition 2. Let $g:(1, \infty) \rightarrow(1, \infty)$ be bijective, continuous and such that

$$
g(x)>x, \quad x>1 .
$$

Then the following conditions are equivalent:

(i) there is a continuous function $f:(1, \infty) \rightarrow(1, \infty)$ such that $C_{f, g}$ is reflexive;

(ii) there is a continuous function $f:(1, \infty) \rightarrow(1, \infty)$ satisfying (5):

$$
f(g(x))=f(x) g(x), \quad x \in(1, \infty) ;
$$

(iii) there are a function $f:(1, \infty) \rightarrow(1, \infty)$ and $c \geq 1$ such that

$$
f(x)=c \prod_{k=0}^{\infty} g^{-k}(x), \quad x \in(1, \infty),
$$

where $g^{-k}$ denotes the $k$ th iterate of the function $g^{-1}$.

Proof. The implication $(i) \Longrightarrow(i i)$ is obvious.

Assume (ii). The assumptions on $g$ imply that $g^{-1}$, the inverse of $g$, is continuous, strictly increasing, and, in view of (6),

$$
1<g^{-1}(x)<x, \quad x>1 .
$$

Consequently,

$$
\lim _{n \rightarrow \infty} g^{-n}(x)=1, \quad x>1,
$$

where $g^{-n}$ stands for the $n$th iterate of $g^{-1}$.

Assume that there is a continuous function $f$ such that equality (5) holds. Replacing $x$ by $g^{-1}(x)$ in (5) we get

$$
f\left(g^{-1}(x)\right)=\frac{f(x)}{x}, \quad x \in(1, \infty),
$$

whence, by induction,

$$
f\left(g^{-n}(x)\right)=\frac{f(x)}{\prod_{k=0}^{n-1} g^{-k}(x)}, \quad x \in(1, \infty), n \in \mathbb{N} .
$$


Since $c:=f(1+) \geq 1$ exists, letting here $n \rightarrow \infty$, we obtain

$$
c=\frac{f(x)}{\prod_{k=0}^{\infty} g^{-k}(x)}, \quad x \in(1, \infty),
$$

whence

$$
f(x)=c \prod_{k=0}^{\infty} g^{-k}(x), \quad x \in(1, \infty),
$$

where the infinite product $\prod_{k=0}^{\infty} g^{-k}$ converges, and its product is a continuous function, which proves that (iii) holds.

Assume (iii). If $f:(1, \infty) \rightarrow(1, \infty)$ is of the form (7) then, for every constant $c \geq 1$ and $x \in(1, \infty)$ we have

$$
\begin{aligned}
f(g(x)) & =c \prod_{k=0}^{\infty} g^{-k}(g(x))=c g(x) \prod_{k=1}^{\infty} g^{-k}(g(x)) \\
& =\left(c \prod_{k=1}^{\infty} g^{-k}\left(g^{1}(x)\right)\right) g(x)=\left(c \prod_{k=0}^{\infty} g^{-k}(x)\right) g(x) \\
& =f(x) g(x) .
\end{aligned}
$$

which implies that $C_{f, g}$ is reflexive, so (i) holds.

Now we prove the main result of this section.

Theorem 3. Let $f, g:(1, \infty) \rightarrow(1, \infty)$ be continuous, strictly increasing and onto. The following conditions are equivalent:

(i) the function $C_{f, g}$ is a bivariable strict mean in $(1, \infty)$;

(ii) the function $C_{f, g}$ is reflexive;

(iii) the infinite product $\prod_{k=0}^{\infty} g^{-k}$ of iterates of $g^{-1}$ converges uniformly on compact subsets of $(1, \infty)$ and,

$$
f=\prod_{k=0}^{\infty} g^{-k}
$$

moreover $C_{f, g}=\mathcal{C}_{g}$, where $\mathcal{C}_{g}:(1, \infty)^{2} \rightarrow(1, \infty)$ defined by

$$
\mathcal{C}_{g}(x, y)=\left(\prod_{k=0}^{\infty} g^{-k+1}\right)^{-1}\left(\prod_{k=0}^{\infty} g^{-k}(x) g(y)\right), \quad x, y>1,
$$

is a strictly increasing bivariable mean in $(1, \infty)$.

Proof. It is obvious that (i) implies (ii). Assume that (ii) holds. Then, applying Propositions 1 and 2 and taking into account that $c:=f(1+)=1$ (by the bijectivity of $f$ ) we get the first part of (iii); in particular the uniform convergence on compact subsets of the infinite product of iterates follows from (7) and the Dini theorem. The "moreover" result follows immediately from (8) and the definition of $C_{f, g}$. Thus (ii) implies (iii).

To prove (i) assuming (iii), note that the function $\mathcal{C}_{g}$ defined by (9) is reflexive and strictly increasing in each variable so, by Remark 2(ii), it is a strict mean.

From the above theorem we obtain the following 
COROllary 1. Let a continuous strictly increasing function $r:(1, \infty) \rightarrow$ $(1, \infty)$ be such that

$$
1<r(x)<x, \quad x>1
$$

and the infinite product

$$
\prod_{k=0}^{\infty} r^{k}
$$

converges to a finite continuous function. Then the function $\mathcal{C}_{r}:(1, \infty)^{2} \rightarrow(1, \infty)$ defined by

$$
\mathcal{C}_{r}(x, y):=\left(\prod_{k=0}^{\infty} r^{k-1}\right)^{-1}\left(\prod_{k=0}^{\infty} r^{k}(x) r^{-1}(y)\right), \quad x, y>1
$$

is a bivariable strict mean in $(1, \infty)$.

PROOF. It is enough to apply the previous result with $g:=r^{-1}, f:=\prod_{k=0}^{\infty} r^{k}$ and observe that $\mathcal{C}_{r}=C_{f, g}$.

It seems to be interesting that the mean $\mathcal{C}_{r}$ is generated with the aid of the iterates of the function $r$ satisfying the assumptions of Corollary 1 .

The mean $\mathcal{C}_{r}$ is constructed with the aid of the iterates of $r$, for convenience, we introduce the following

Definition 1. If $r:(1, \infty) \rightarrow(1, \infty)$ satisfies the assumptions of Corollary 1 , then the function $\mathcal{C}_{r}$ is called a product iterative mean of generator $r$.

EXAMPLE 1. Applying the definition of $\mathcal{C}_{r}$ for the generator $r:(1, \infty) \rightarrow(1, \infty)$ given by $r(x)=x^{w}$, where $w \in(0,1)$ is fixed, we get weighted geometric mean $\mathcal{C}_{r}(x, y)=x^{w} y^{1-w}$ for all $x, y \in(1, \infty)$.

REMARK 4. In Theorem 2 we assume that $I=(1, \infty)$. One could also get the suitable results if $I$ is one the following intervals $[1, \infty),(0,1),(0,1]$, and $(0, \infty)$.

\section{Invariant operation with respect to iterative mean-type mapping need not be a mean}

In this section we focus our attention on the question whether the invariant function $C_{f \circ g, g \circ h}$ occurring in the following counterpart of (1):

$$
C_{f \circ g, g \circ h} \circ\left(C_{f, g}, C_{g, h}\right)=C_{f \circ g, g \circ h},
$$

is a mean, if the coordinates of the mapping $\left(C_{f, g}, C_{g, h}\right)$ defined by $(2)$ are means.

If $C_{f, g}=\mathcal{C}_{g}$ and $C_{g, h}=\mathcal{C}_{h}$, then, in view of [7, Theorem 1], the function $C_{f \circ g, g \circ h}$ is invariant with respect to the mean type mapping $\left(\mathcal{C}_{g}, \mathcal{C}_{h}\right)$.

On the other hand $\mathcal{C}_{g}$ (and $\mathcal{C}_{h}$ ) is a very special case of generalized weighted quasi-geometric mean $G_{\varphi, \psi}: I^{2} \rightarrow I$,

$$
G_{\varphi, \psi}(x, y)=(\varphi \psi)^{-1}(\varphi(x) \psi(y)), \quad x, y \in I,
$$

where $\varphi, \psi: I \rightarrow(0, \infty)$. Indeed, with $\varphi=\prod_{k=0}^{\infty} g^{-k}$ and $\psi=g$ we have $G_{\varphi, \psi}=\mathcal{C}_{g}$ (and with $\psi=\prod_{k=0}^{\infty} h^{-k}$ and $\gamma=h$ we have $G_{\psi, \gamma}=\mathcal{C}_{h}$ ). Moreover, according to [5] and Remark 3] if $G_{\varphi, \psi}$ and $G_{\psi, \gamma}$ are two generalized weighted quasi-geometric 
means, then $G_{\varphi \psi, \psi \gamma}$, the mean of the same type, is a unique mean that is invariant with respect to the mean-type mapping $\left(G_{\varphi, \psi}, G_{\psi, \gamma}\right): I^{2} \rightarrow I^{2}$.

In this context the question arises whether the invariant function $C_{f \circ g, g \circ h}$ coincides with the invariant mean $G_{\varphi \psi, \psi \gamma}$ ? In view of Remark 3 and ([5]), the answer is positive, if $C_{f \circ g, g \circ h}$ is a mean, i.e. if there is a suitable bijective function $u:(1, \infty) \rightarrow(1, \infty)$ such that $C_{f \circ g, g \circ h}=\mathcal{C}_{u}$.

We prove

Theorem 4. Assume that $f, g, h:(1, \infty) \rightarrow(1, \infty)$ are continuous, strictly increasing, and onto. Then $C_{f, g}, C_{g, h}$, and $C_{f \circ g, g \circ h}$ are means in $(1, \infty)$, iff

$$
g=\prod_{i=0}^{\infty} h^{-i}, \quad f=\prod_{j=0}^{\infty}\left(\prod_{i=0}^{\infty} h^{-i}\right)^{-j},
$$

and $h$ satisfies the composite functional equation

$$
\prod_{j=0}^{\infty}\left(\prod_{i=0}^{\infty} h^{-i}\right)^{-j+1}=\prod_{k=0}^{\infty}\left(\prod_{i=0}^{\infty} h^{-i+1}\right)^{-k},
$$

where $i, j, k$ stand for the indices of iterates of the suitable functions.

Proof. Assume that $C_{f, g}, C_{g, h}$ and $C_{f \circ g, g \circ h}$ are means in $(1, \infty)$. In view of Theorem 3, we have $C_{g, h}=\mathcal{C}_{h}, C_{f, g}=\mathcal{C}_{g}, C_{f \circ g, g \circ h}=\mathcal{C}_{g \circ h}$, and

$$
g=\prod_{i=0}^{\infty} h^{-i}, \quad f=\prod_{j=0}^{\infty} g^{-j}, \quad f \circ g=\prod_{k=0}^{\infty}(g \circ h)^{-k} .
$$

It follows that (10) holds true, and the third of equalities (12) implies that $h$ satisfies the composite functional equation,

$$
\left(\prod_{j=0}^{\infty}\left(\prod_{i=0}^{\infty} h^{-i}\right)^{-j}\right) \circ\left(\prod_{i=0}^{\infty} h^{-i}\right)=\prod_{k=0}^{\infty}\left(\left(\prod_{i=0}^{\infty} h^{-i}\right) \circ h\right)^{-k},
$$

which simplifies to (11). The converse implication is obvious.

Thus our question leads to rather complicate composite functional equation (11). We pose the following

Problem 1. Determine strictly increasing bijective functions $h:(1, \infty) \rightarrow$ $(1, \infty)$ satisfying equation (11).

Let us consider the following

Example 2. Take $w \in(0,1)$, and define $h:(1, \infty) \rightarrow(1, \infty)$ by $h(x)=x^{\frac{1}{w}}$. From Theorem 3 with $g$ and $f$ replaced, respectively, by $h$ and $g$, we get

$$
g(x)=\prod_{k=0}^{\infty} h^{-k}(x)=\prod_{k=0}^{\infty} x^{w^{k}}=x^{\frac{1}{1-w}}, \quad x \in(1, \infty),
$$

and, for all $x, y>1$,

$$
\begin{aligned}
\mathcal{C}_{h}(x, y) & =\left(\prod_{k=0}^{\infty} h^{-k+1}\right)^{-1}\left(\prod_{k=0}^{\infty} h^{-k}(x) h(y)\right) \\
& =\left(x^{\frac{1}{1-w}} y^{\frac{1}{w}}\right)^{w(1-w)}=x^{w} y^{1-w} .
\end{aligned}
$$


Similarly, as $g^{-1}(x)=x^{1-w}$, we get

$$
f(x)=\prod_{k=0}^{\infty} g^{-k}(x)=\prod_{k=0}^{\infty} x^{(1-w)^{k}}=x^{\frac{1}{w}}, \quad x \in(1, \infty),
$$

and, for all $x, y>1$,

$$
\begin{aligned}
\mathcal{C}_{g}(x, y) & =\left(\prod_{k=0}^{\infty} g^{-k+1}\right)^{-1}\left(\prod_{k=0}^{\infty} g^{-k}(x) g(y)\right) \\
& =\left(x^{\frac{1}{w}} y^{\frac{1}{1-w}}\right)^{w(1-w)}=x^{1-w} y^{w} .
\end{aligned}
$$

Moreover, since $(f \circ g)(x)=x^{\frac{1}{w(1-w)}},(g \circ h)(x)=x^{\frac{1}{(1-w) w}}$, we get, for all $x, y>$ 1 ,

$$
\begin{aligned}
C_{f \circ g, g \circ h}(x, y) & =((f \circ g) \circ(g \circ h))^{-1}((f \circ g)(x) \cdot(g \circ h)(y)) \\
& =\left(x^{\frac{1}{w(1-w)}} y^{\frac{1}{(1-w) w}}\right)^{w^{2}(1-w)^{2}}=(x y)^{w(1-w)},
\end{aligned}
$$

whence

$$
C_{f \circ g, g \circ h}=\mathcal{G}^{2 w(1-w)},
$$

where $\mathcal{G}(x, y)=\sqrt{x y}(x, y>1)$ is the geometric mean.

For every $w \in(0,1)$ we have $\mathcal{G} \circ\left(\mathcal{C}_{g}, \mathcal{C}_{h}\right)=\mathcal{G}$, and $\mathcal{G}$ is a unique $\left(\mathcal{C}_{g}, \mathcal{C}_{h}\right)$ invariant mean. The function $C_{f \circ g, g \circ h}$ is also invariant for every $w \in(0,1)$, but $C_{f \circ g, g \circ h}$ is not a mean for any $w \in(0,1)$.

In the context of the above discussion let us consider the following

REMARK 5. Under conditions of Theorem 4, the bijective functions $f, g, h$ : $(1, \infty) \rightarrow(1, \infty)$ are increasing. Consequently, they are almost everywhere differentiable and $f(1+)=g(1+)=h(1+)=1$. Assume additionally that $f(1)=g(1)=$ $h(1)=1$ and that $f, g, h$ are differentiable at the point 1 . If the functions $C_{f, g}$, $C_{g, h}$ and $C_{f \circ g, g \circ h}$ were means in $[1, \infty)$ then, by their reflexivity, we would have

$f(g(x))=f(x) g(x), \quad g(h(x))=g(x) h(x), \quad f\left(g^{2}(h(x))\right)=f(g(x)) g(h(x))$,

for all $x \geq 1$, where $g^{2}$ is the second iterate of $g$. Differentiating both sides of each of these equalities at $x=1$ and then setting

$$
a:=f^{\prime}(1), \quad b:=g^{\prime}(1), \quad c:=h^{\prime}(1),
$$

we would get

$$
a b=a+b, \quad b c=b+c, \quad a b^{2} c=a b+b c .
$$

Since this system has no solution satisfying the conditions $a \geq 1, b \geq 1, c \geq 1$, the functions $f, g, h$ do not exist.

\section{Example of application of invariance identity}

In this section we show that knowing the invariant mean with respect to a given continuous and strict mean-type mapping we can determine effectively the limit of the sequence of its iterates, as well as, to find the form of all continuous functions which are invariant with respect to this mapping. 
TheOREM 5. Let $f, g:(0, \infty) \rightarrow(0, \infty)$ be continuous strictly increasing functions such that the function $\frac{f}{g}$ is strictly increasing. Then

(i) the functions $M, N:(0, \infty)^{2} \rightarrow(0, \infty)$ given by

$$
M(x, y):=f^{-1}\left(\frac{g(y)}{g(x)} f(x)\right), \quad N(x, y):=f^{-1}\left(\frac{g(x)}{g(y)} f(y)\right)
$$

are generalized weighted quasi-geometric means, as $M=G_{\frac{f}{g}, g}$ and $N=G_{g, \frac{f}{g}}$;

(ii) the quasi-geometric mean $G_{f}:(0, \infty)^{2} \rightarrow(0, \infty)$ given by

$$
G_{f}(x, y)=f^{-1}(\sqrt{f(x) f(y)})
$$

is invariant with respect to the mean-type mapping $(M, N)$, i.e.

$$
G_{f} \circ(M, N)=G_{f}
$$

(iii) the sequence $\left((M, N)^{n}: n \in \mathbb{N}\right)$ of iterates of the mean-type mapping $(M, N)$ : $(0, \infty)^{2} \rightarrow(0, \infty)^{2}$ converges uniformly on compact subsets of $(0, \infty)^{2}$ and

$$
\lim _{n \rightarrow \infty}(M, N)^{n}=\left(G_{f}, G_{f}\right) ;
$$

(iv) a continuous function $\Phi:(0, \infty)^{2} \rightarrow \mathbb{R}$ is invariant with respect to the mean-type mapping $(M, N)$, i.e. $\Phi$ satisfies the equation

$$
\Phi(M(x, y), N(x, y))=\Phi(x, y), \quad x, y>0,
$$

if and only if there is a continuous single variable function $\varphi:(0, \infty) \rightarrow \mathbb{R}$ such that

$$
\Phi(x, y)=\varphi\left(G_{f}(x, y)\right), \quad x, y>0 .
$$

Proof. (i) By assumption, $M$ and $N$ are both reflexive and strictly increasing in each variable so, by Remark 2(ii), they are strict means. The remaining result is easy to verify.

(ii) By the definitions of $G_{f}, M$ and $N$, we have, for all $x, y>0$,

$$
\begin{aligned}
G_{f} \circ(M, N)(x, y) & =G_{f}((M(x, y), N(x, y))) \\
& =f^{-1}\left(\sqrt{f\left[f^{-1}\left(\frac{g(y)}{g(x)} f(x)\right)\right] \cdot f\left[f^{-1}\left(\frac{g(x)}{g(y)} f(y)\right)\right]}\right) \\
& =f^{-1}(\sqrt{f(x) f(y)})=G_{f}(x, y),
\end{aligned}
$$

which proves (ii).

Since $M$ and $N$ are strict and continuous means, (iii) follows from (ii) and the result of [2] (see also [3]).

(iv) Assume that $\Phi:(0, \infty)^{2} \rightarrow \mathbb{R}$ is invariant with respect to the mean-type mapping $(M, N)$, so

$$
\Phi(x, y)=\Phi(M(x, y), N(x, y))=(\Phi \circ(M, N))(x, y), \quad x, y>0 .
$$

Hence, by induction,

$$
\Phi(x, y)=\left(\Phi \circ(M, N)^{n}(x, y)\right), \quad n \in \mathbb{N} ; x, y>0 .
$$

Hence, in view of (iii), by the continuity of $\Phi$, we have, for all $x, y>0$,

$$
\begin{aligned}
\Phi(x, y) & =\lim _{n \rightarrow \infty}\left(\Phi \circ(M, N)^{n}(x, y)\right)=\left(\Phi \circ\left(G_{f}, G_{f}\right)\right)(x, y) \\
& =\Phi\left(G_{f}(x, y), G_{f}(x, y)\right)=\varphi\left(G_{f}(x, y)\right),
\end{aligned}
$$


where $\varphi:(0, \infty) \rightarrow \mathbb{R}$ is defined by

$$
\varphi(x):=\Phi(x, x), \quad x>0 .
$$

To prove the converse implication, take an arbitrary function $\varphi:(0, \infty) \rightarrow \mathbb{R}$ and define $\Phi:(0, \infty)^{2} \rightarrow \mathbb{R}$ by

$$
\Phi(x, y):=\varphi\left(G_{f}(x, y)\right) .
$$

Then, for arbitrary $x, y>0$, using the definition of $\Phi$ and (ii) we get

$$
(\Phi \circ(M, N))(x, y)=\varphi\left(G_{f}(M(x, y), N(x, y))\right)=\varphi\left(G_{f}(x, y)\right)=\Phi(x, y),
$$

which proves $(\mathrm{v})$.

REMARK 6. Let $f, g, h: I \rightarrow(0, \infty)$ be continuous and all strictly increasing or all strictly decreasing. Using a similar argument to the proof of Theorem 5 , we can show that a continuous function $\Phi: I^{2} \rightarrow \mathbb{R}$ is invariant with respect to the mean-type mapping $\left(G_{f, g}, G_{g, h}\right): I^{2} \rightarrow I$ if and only if there is a continuous single variable function $\varphi: I \rightarrow \mathbb{R}$ such that $\Phi(x, y)=\varphi\left(G_{f g, g h}(x, y)\right)$ for all $x, y \in I$.

A similar argument to the proof of Theorem [5 shows the following

COROLlaRY 2. Let $f, g: \mathbb{R} \rightarrow \mathbb{R}$ be continuous strictly increasing functions such that the function $f-g$ is strictlay increasing. Then

(i) The functions $M, N: \mathbb{R}^{2} \rightarrow \mathbb{R}$ given by

$$
M(x, y):=f^{-1}(g(y)-g(x)+f(x)), \quad N(x, y):=f^{-1}(g(x)-g(y)+f(y))
$$

are generalized weighted strict quasi-arithmetic means, as $M=A_{f-g, g}$ and $N=$ $A_{g, f-g}$

(ii) the quasi-arithmetic mean $A_{f}: \mathbb{R}^{2} \rightarrow \mathbb{R}$ given by

$$
A_{f}(x, y)=f^{-1}\left(\frac{f(x)+f(y)}{2}\right)
$$

is invariant with respect to the mean-type mapping $(M, N)$, i.e.

$$
A_{f} \circ(M, N)=A_{f}
$$

(iii) the sequence $\left((M, N)^{n}: n \in \mathbb{N}\right)$ of iterates of the mean-type mapping $(M, N)$ : $\mathbb{R}^{2} \rightarrow \mathbb{R}^{2}$ converges uniformly on compact subsets of $\mathbb{R}^{2}$ and

$$
\lim _{n \rightarrow \infty}(M, N)^{n}=\left(A_{f}, A_{f}\right) ;
$$

(iv) a continuous function $\Phi: \mathbb{R}^{2} \rightarrow \mathbb{R}$ is invariant with respect to the meantype mapping $(M, N)$, i.e. $\Phi$ satisfies the equation

$$
\Phi(M(x, y), N(x, y))=\Phi(x, y), \quad x, y \in \mathbb{R},
$$

if and only if there is a continuous single variable function $\varphi: \mathbb{R} \rightarrow \mathbb{R}$ such that

$$
\Phi(x, y)=\varphi\left(A_{f}(x, y)\right), \quad x, y \in \mathbb{R} .
$$




\section{Equality of two considered operations}

Let $I \subset(0, \infty)$ be an interval that is closed with respect to the addition and multiplication. In this section, given two operations $C_{f, g}$ and $C_{\phi, \psi}$ (resp. $D_{f, g}$ and $\left.D_{\bar{f}, \bar{g}}\right)$, we determine the conditions under which they are equal.

Proposition 3. Let $f, g, \phi, \psi: I \rightarrow I$ be bijective and continuous functions. Then we have $C_{f, g}=C_{\phi, \psi}$ iff $g=\psi$ and $f=a \phi$, for some $a \in I, a \neq 0$.

Proof. (Necessity) Suppose that

$$
C_{f, g}(x, y)=C_{\phi, \psi}(x, y) \quad x, y \in I,
$$

that is,

$$
(f \circ g)^{-1}(f(x) g(y))=(\phi \circ \psi)^{-1}(\phi(x) \psi(y)) \quad x, y \in I .
$$

Setting $\alpha:=(f \circ g) \circ(\phi \circ \psi)^{-1}, \beta:=f \circ \phi^{-1}, \gamma:=g \circ \psi^{-1}, u=\phi(x)$, and $v=\psi(y)$ we get

$$
\alpha(u v)=\beta(u) \gamma(v) \quad u \in \phi(I), v \in \psi(I) .
$$

The latter equation is Pexider's functional equation and has the following solutions (see, e.g., [1])

$$
\beta(u)=a u^{c} \quad \text { and } \quad \gamma(v)=b v^{c} \quad u \in \phi(I), v \in \psi(I),
$$

for some $a, b, c \in I, a, b, c \neq 0$. From these equations we get

$$
f(x)=a \phi(x)^{c} \text { and } g(y)=b \psi(y)^{c} \quad x, y \in I,
$$

for some $a, b, c \in I, a, b, c \neq 0$. Thus, on the one hand, we get

$$
(f \circ g)^{-1}(f(x) g(y))=\psi^{-1}\left(\left(\frac{1}{b} \phi^{-1}\left(\left(\phi(x)^{c} b \psi(y)^{c}\right)^{\frac{1}{c}}\right)\right)^{\frac{1}{c}}\right) \quad x, y \in I,
$$

for some $b, c \in I, b, c \neq 0$, and on the other hand, we get

$$
(\phi \circ \psi)^{-1}(\phi(x) \psi(y))=\psi^{-1}\left(\phi^{-1}(\phi(x) \psi(y))\right) \quad x, y \in I .
$$

Hence, we get

$$
\psi^{-1}\left(\left(\frac{1}{b} \phi^{-1}\left(\left(\phi(x)^{c} b \psi(y)^{c}\right)^{\frac{1}{c}}\right)\right)^{\frac{1}{c}}\right)=\psi^{-1}\left(\phi^{-1}(\phi(x) \psi(y))\right) \quad x, y \in I,
$$

which implies that $b=c=1$. Thus, for all $x \in I$, we get $g(x)=\psi(x)$ and $f(x)=a \phi(x)$, for some $a \in I, a \neq 0$.

(Sufficiency) Obvious.

Proposition 4. Let $f, g, \bar{f}, \bar{g}: I \rightarrow I$ be bijective and continuous functions. Then we have $D_{f, g}=D_{\bar{f}, \bar{g}}$ iff $f=\bar{f}+a$, for some $a \in I$, and $g=\bar{g}$.

Proof. (Necessity) Suppose that

$$
D_{f, g}(x, y)=D_{\bar{f}, \bar{g}}(x, y), \quad x, y \in I,
$$

that is,

$$
(f \circ g)^{-1}(f(x)+g(y))=(\bar{f} \circ \bar{g})^{-1}(\bar{f}(x)+\bar{g}(y)), \quad x, y \in I .
$$

Setting $\alpha:=(f \circ g) \circ(\bar{f} \circ \bar{g})^{-1}, \beta:=f \circ(\bar{f})^{-1}, \gamma:=g \circ(\bar{g})^{-1}, u=\bar{f}(x)$, and $v=\bar{g}(y)$ we get

$$
\alpha(u+v)=\beta(u)+\gamma(v), \quad u \in \bar{f}(I), v \in \bar{g}(I) .
$$


The latter equation is Pexider's functional equation and has the following solutions (see, e.g., [1])

$$
\beta(u)=a u+b \quad \text { and } \quad \gamma(v)=a v+c, \quad u \in \bar{f}(I), v \in \bar{g}(I),
$$

for some $a, b, c \in I, a \neq 0$. From these equations we get

$$
f(x)=a \bar{f}(x)+b \quad \text { and } \quad g(y)=a \bar{g}(y)+c, \quad x, y \in I,
$$

for some $a, b, c \in I, a \neq 0$. Thus, on the one hand, we get

$$
(f \circ g)^{-1}(f(x)+g(y))=(\bar{g})^{-1}\left(\frac{(\bar{f})^{-1}\left(\bar{f}(x)+\bar{g}(y)+\frac{c}{a}\right)-c}{a}\right), \quad x, y \in I,
$$

for some $a, c \in I, a \neq 0$, and on the other hand, we get

$$
(\bar{f} \circ \bar{g})^{-1}(\bar{f}(x)+\bar{g}(y))=(\bar{g})^{-1}\left((\bar{f})^{-1}(\bar{f}(x)+\bar{g}(y))\right), \quad x, y \in I .
$$

Hence, we get

$$
(\bar{g})^{-1}\left(\frac{(\bar{f})^{-1}\left(\bar{f}(x)+\bar{g}(y)+\frac{c}{a}\right)-c}{a}\right)=(\bar{g})^{-1}\left((\bar{f})^{-1}(\bar{f}(x)+\bar{g}(y))\right), \quad x, y \in I,
$$

which implies that $a=1$ and $c=0$. Thus, for all $x \in I$, we get $f(x)=\bar{f}(x)+b$, for some $b \in I$, and $g(x)=\bar{g}(x)$.

(Sufficiency) Obvious.

\section{Acknowledgements}

This research is partly supported by the internal research project R-AGR-0500 of the University of Luxembourg and by the Luxembourg National Research Fund R-AGR-3080.

\section{References}

[1] J. Aczél. Lectures in Functional Equations and Their Applications, Dover Publications, Inc., New York, 2006.

[2] J. Matkowski, Iterations of mean-type mappings and invariant means, Ann. Math. Siles. 13 (1999), 211-226.

[3] J. Matkowski, Iterations of the mean-type mappings, (ECIT' 08), (A.N. Sharkovsky, I.M. Sushko (Eds.), Grazer Math. Ber., Bericht Nr. 354 (2009), 158-179.

[4] J. Matkowski. Generalized weighthed quasi-arithmetic means. Aeq. Math., 79:203-212, 2010.

[5] J. Matkowski. Iterations of mean-type mappings and uniqueness of invariant means. Ann. Univ. Sci. Budapest, 41:145-158, 2013.

[6] J. Matkowski. Invariance identity in the class of generalized quasiarithmetic means. Colloquium Mathematicum, vol. 137, No. 2, 2014.

[7] J. Devillet and J. Matkowski. A new invariance identity and means. arXiv: 1807.04811.

Current address: Mathematics Research Unit, University of Luxembourg, Maison du Nombre, 6, avenue de la Fonte, L-4364 Esch-sur-Alzette, Luxembourg

E-mail address: jimmy.devillet@uni.lu

Current address: Faculty of Mathematics Computer Science and Econometrics, Univerity of Zielona Góra, Szafrana 4A, PL 65-516 Zielona Góra, Poland

E-mail address: J.Matkowski@wmie.uz.zgora.pl 